\title{
Vollzug und Sprache Physischer Geographie und die Frage geographischen Takts
}

\author{
B. Zahnen \\ Geographisches Institut, Humboldt-Universität zu Berlin, Unter den Linden 6, 10099 Berlin, Germany \\ Received: 3 February 2011 - Published in Soc. Geogr. Discuss.: 10 February 2011 \\ Revised: 29 April 2011 - Accepted: 27 May 2011 - Published: 1 June 2011
}

\begin{abstract}
Zusammenfassung. Der Aufsatz versteht sich als ein Beitrag zu der sozial- oder kulturwissenschaftlichen Diskussion der Humangeographie um die Möglichkeit der Einbeziehung einer Dimension der Natur bzw. der „Materialität“, zugleich aber auch als ein theoretischer Entwurf einer „Physischen Geographie im starken Sinne“, die nicht mehr in dichotomer Entgegensetzung zur Humangeographie zu verstehen ist und die Möglichkeit bietet, genuin geographische Prozesse und Seinsweisen als solche zu verstehen.
\end{abstract}

Ausgehend davon, dass es Physische Geographen bzw. Geowissenschaftler gibt, die ihr Tun als „Gespräch mit der Erde“ bezeichnen, deckt der Aufsatz zum einen auf, inwiefern dieses Tun aufgrund einer grundlegenden Ebene, die sich aus dem Prozess der eigenen Erfahrung übergänglicher Naturgebilde der Erde ergibt, als etwas qualifiziert werden kann, das eine gewisse Form „doppelter Hermeneutik“ involviert. Zum anderen wird gezeigt, dass aufgrund dieser grundlegenden Ebene die Möglichkeit des revidierten Verständnisses von Physischer Geographie - also der „Physischen Geographie im starken Sinne“ - in dem derzeitigen physisch-geographischen Tun eines naturwissenschaftlichen Selbstverständnisses bereits angelegt ist. Ferner wird deutlich, dass sich in diesem Zuge neue Zugangsmöglichkeiten zur Geschichte des Fachs ergeben. In diesem Sinne wird die alte Idee eines ,geographischen Takts“ neu beleuchtet und entfaltet.

\begin{abstract}
The paper can be understood as a contribution to the discussion about how to involve "nature" or "materiality" in the undertakings of social or cultural scientists or human geographers, but at the same time as a theoretical conception of "physical geography in a strong sense" which can no longer be understood as being in dichotomic opposition to human geography and makes it possible to understand genuinely geographical processes and qualities [Seinsweisen] as such.

Taking as a starting point the fact that there are physical geographers and earth scientists who characterise their doing as a "conversation with the earth", the paper reveals in which way it is appropriate to qualify this doing as involving a kind of "double hermeneutics": namely due to a foundational layer which arises from the process of experiencing transitional formations of the natural appearances of the earth. The paper also shows that due to this foundational layer, the chance of a revised understanding of physical geography i.e. of "physical geography in the strong sense" - is already inherent in current physical geography as a natural science. Moreover, the paper shows that its insights open up new ways to understand pivotal traits of the history of geography. In this sense, the old idea of a "geographical tact" is illuminated and unfolded in a new way.
\end{abstract}

\section{Einführung}

Der vorliegende Aufsatz thematisiert physisch-geographisches Tun aus der Sicht und der Erfahrung einer Physischen Geographin und ist doch - trotzdem oder gerade deswegen - als ein Beitrag zu der humangeographischen

Correspondence to: B. Zahnen

(barbara.zahnen@geo.hu-berlin.de) (bzw. sozial- oder kulturwissenschaftlichen) Diskussion zu verstehen, die sich darum dreht, wie eigentlich das, was derzeit meist ,materielle Dimension“ genannt wird, in unser Denken und somit auch unsere Darstellungen einbezogen werden kann. Ich verstehe ebendiese Frage so, dass es dabei um so etwas wie eine Welt der Naturerscheinungen geht, die sich nicht auf Gesellschaftliches, „soziale Konstruktionen“, Kommunikationsprozesse oder eine bloße Welt der Zeichen, Diskurse und Begriffe reduzieren lässt, obwohl wir 
Menschen es natürlich sind und sein müssen, die diese Naturerscheinungen wahrnehmen und thematisch machen. Der vorliegende Aufsatz sucht aufzuzeigen, dass man in Bezug auf die genannte Frage - und somit auch in Bezug auf die Frage eines „Mensch-Natur-Verhältnisses“ - eben nicht unbedingt auf Ansätze zurückgreifen muss, die in den sogenannten „Nachbarwissenschaften“ der Geographie, etwa den Sozial- oder Kulturwissenschaften, entwickelt wurden, sondern einiges von bzw. anhand der Physischen Geographie selbst lernen kann. Und dies gerade dann, wenn man - wie ich im Zuge dieses Aufsatzes - auf etwas stößt, in dem ich so etwas wie einen „brachliegenden Kern“ der Physischen Geographie sehe: Dabei spreche ich von „Kern“ zum einen deswegen, weil es sich um Grundzüge handelt, ohne die die Physische Geographie, auch in ihrer derzeitigen naturwissenschaftlichen Ausprägung, nicht ernsthaft auskommt, und zum anderen, weil sich auf der Basis dieser Grundzüge eine eigene (jedoch nicht essenzialistisch zu verstehende) Identität der Physischen Geographie im Unterschied zu anderen „Geowissenschaften“ oder ,erdbezogenen Naturwissenschaften" (also der Geologie, Bodenkunde, Meteorologie etc.) herauszubilden vermag. „Brachliegend“ bezeichne ich diesen Kern deswegen, weil er bisher mehr im Impliziten liegt, als dass er explizit als eigenes Potenzial verstanden worden wäre, und weil er für die gegenwärtige Physische Geographie ein neues Selbstverständnis, eine revidierte Form physisch-geographischen Arbeitens bedeuten könnte. Diese möchte ich als „Physische Geographie im starken Sinne"1 bezeichnen. Ihr Charakteristikum liegt - wie ich zeigen möchte - darin, dass sie genuin geographische Prozesse oder Seinsweisen als solche verstehbar werden bzw. zur Darstellung kommen lässt. Zudem bedeutet diese Physische Geographie im starken Sinne auch, dass eine dichotome Entgegensetzung von Physischer Geographie und Humangeographie hinfällig wird. Vor diesem Hintergrund gilt der Aufsatz als ein Beitrag zur Humangeographie wie zur Physiogeographie gleichermaßen. Weil die Fragestellung aber auch das Verhältnis zu den „Nachbarwissenschaften“ der Geographie betrifft, weist sie auch über die Geographie hinaus.

${ }^{1}$ Es würde hier zu weit führen, genau zu erläutern, warum ich bewusst nicht die Formulierung einer Physischen Geographie im engeren (statt weiteren) Sinne gewählt habe, sondern von einer Physischen Geographie im starken Sinne spreche. Die auf den ersten Blick eingängigere Formulierung einer Physischen Geographie im engeren Sinne würde zu Problemen führen, wenn ich an anderer Stelle weitere Aspekte der im vorliegenden Aufsatz behandelten Problematik ausführe. Abgesehen davon passt zu der von mir präferierten Formulierung, dass in der Wissenschaftsphilosophie zwischen einer schwachen und starken Hermeneutik der Naturwissenschaften unterschieden wird, wobei letztere, wie eben auch der vorliegende Aufsatz, von der hermeneutischen Philosophie bzw. hermeneutischen Phänomenologie Heideggers inspiriert ist (vgl. Heelan, 1989; Ginev, 2002).
Es mag nun irritieren, dass im Folgenden einerseits von etwas gesprochen werden soll, das sich - wie gesagt nicht auf soziale Konstruktionen, Kommunikationsprozesse, Zeichen und Diskurse etc. reduzieren lässt, andererseits aber im Titel ausgerechnet von der „Sprache Physischer Geographie“ die Rede ist. Mit der Formulierung „Sprache Physischer Geographie“" geht es mir jedoch nicht allein darum, dass wir uns über die Erfahrung von Naturerscheinungen der Erde auch irgendwie sprachlich verständigen müssen. Vielmehr möchte ich noch einen Schritt weiter gehen und etwas behaupten, das mit humangeographischen Ansätzen der letzten Jahrzehnte, und zwar insbesondere mit poststrukturalistischen Positionen inkompatibel zu sein scheint: nämlich dass es so etwas wie eine Sprache der Dinge, hier: eine Sprache der irdischen Naturerscheinungen selbst gibt. In ähnlicher Weise spricht man umgangssprachlich ja auch davon, dass die Dinge für sich selbst sprechen oder - im Falle eines Irrtums - dass die Dinge eine andere Sprache sprechen. Ich verstehe daher die „Sprache Physischer Geographie“ im zweifachen Sinne: eben nicht allein im Sinne einer Sprache, die Physische Geographinnen und Geographen sprechen, sondern eben auch im Sinne einer Sprache dessen, was man den Gegenstand der physisch-geographischen Bemühungen nennen könnte. Dass der Begriff Geographie sich eben nicht nur auf ein Fach bzw. ein Produkt menschlicher Tätigkeit bezieht, sondern auch auf den physisch verorteten Gegenstand der Bemühungen dieses Fachs, war ja zu früheren Zeiten durchaus üblich.

Wenn nun im Titel nicht nur von Sprache, sondern zugleich auch noch von Vollzug die Rede ist, dann aufgrund der zentralen These, dass sich das eben angesprochene Problem der Sprache Physischer Geographie in ihrem zweifachen Sinn - und mit diesem auch die von mir postulierte Physische Geographie im starken Sinne - daraus verstehen lässt, wie sich Physische Geographie vollzieht. Dass hier von „Vollzug“ - statt in aktueller Manier von „Performanz“ oder „Performativität" - gesprochen wird, erklärt sich aus dem hermeneutisch-phänomenologischen Hintergrund der Überlegungen. Darauf, wie dieser Hintergrund - ein durch Heidegger ermöglichtes, aber auch in Gadamers Philosophie Niederschlag findendes Denken - einzuordnen ist, werde ich im folgenden Abschnitt zurückkommen.

\section{2 "Gespräch mit der Erde“"}

Zunächst komme ich zu einem interessanten Phänomen, das in einem Bezug zu der eben genannten zentralen These steht und sich in der jüngeren physisch-geographischen bzw. geologischen Literatur findet: Dort ist nämlich, z.B. bei Phillips (1999:759) und bei Baker (2000) - beides sind sehr renommierte und erfahrene Naturwissenschaftler -, von einem „Gespräch mit der Erde“ die Rede, und diese Formulierung wird gerade dazu verwendet, um mit ihr das wissenschaftliche Tun der Physischen Geographie bzw. 
der Geologie zu charakterisieren. Mit der Formulierung „Gespräch mit der Erde“ bezieht sich zumindest Baker auf den gleichlautenden Titel eines Buches von Hans Cloos (1947, hier 1968), einem 1951 verstorbenen ebenfalls sehr angesehenen Geologen, der in seinem Buch sogar vom „Zwiegespräch“ mit der Erde spricht, das er im Laufe seines wissenschaftlichen Lebens gehalten hat (vgl. ebd., 13). Nun setzt eine Art Zwiegespräch mit der Erde auch eine Art Sprache der Erde voraus, und wenn hier erörtert werden soll, inwiefern sich das Problem der Sprache Physischer Geographie in dem oben genannten zweifachen Sinne daraus verstehen lässt, wie sich Physische Geographie vollzieht, dann eben indem diese Idee des "Gesprächs“ bzw. „Zwiegesprächs“ mit der Erde vertieft wird - und zwar auf einen für die Physische Geographie wesentlichen Aspekt hin, den man meines Erachtens bisher übersehen hat.

Denn auch Demeritt und Dyer (2002), die die Rolle des Dialogs oder von Metaphern des Dialogs in Forschungspraktiken bzw. zur Beschreibung von Forschungspraktiken von Human- und Physischer Geographie diskutieren, haben sich damit beschäftigt, dass Physische Geographen oder Geologen für ihr Tun die Metapher eines Gesprächs mit der Erde verwenden. Aber ohne dass auf diesen Aufsatz hier im Detail eingegangen werden kann, ist doch Folgendes herauszustellen:

Demeritt und Dyer bringen die Begriffe „dialogue“ bzw. ,conversation“ in ihrem Bezug auf wissenschaftliches Tun mit der hermeneutischen Tradition in Verbindung, argumentieren dabei aber vor allem mit der Diltheyschen Hermeneutik und somit im Sinne einer Hermeneutik, die stark dadurch motiviert war, die Geisteswissenschaften methodisch von den Naturwissenschaften abzugrenzen. Zwar wird auch die Heideggersche Hermeneutik angesprochen - jedoch nur in Form eines Ausblicks, der Alternativen zu der von den Autoren analysierten Fixierung auf das Dialogische darzustellen sucht - was meines Erachtens der Heideggerschen Hermeneutik wie auch dem Phänomen des Gesprächs keineswegs gerecht wird. Es klingt auch eine mittelbare Rezeption von Aspekten der Gadamerschen Hermeneutik durch: nicht nur etwa da, wo die an das Gadamersche Konzept der Vorurteilshaftigkeit erinnernden „preconceptions“ (ebd., 234) erwähnt werden, die in Forschungspraktiken in Frage zu stellen seien, sondern vor allem durch die gedankliche Zusammenführung von Hermeneutik einerseits und dem Phänomen des Gesprächs andererseits, die in Gadamers Philosophie ja eine wesentliche Rolle spielt (vgl. Gadamer, 1999c). Doch was Demeritt und Dyer nicht bewusst zu sein scheint, ist, dass die durch Heidegger ermöglichte neuere Hermeneutik das Potenzial birgt, Gemeinsamkeiten zwischen natur- und humanwissenschaftlichen Forschungspraktiken bzw. zwischen Physischer Geographie und Humangeographie zur Sprache zu bringen, die über methodische Fragen hinausgehen, weil sie tieferliegend - die menschliche Existenz betreffen, ohne dabei ein „bloß philosophisches“ und somit für die Praxis der Fachwissenschaften unbedeutsames Thema zu sein, wie es Demeritt und Dyer (2002:237) der Heideggerschen Hermeneutik unterstellen.

Vor dem Hintergrund dieses blinden Flecks ist es völlig verständlich, dass und inwiefern Demeritt und Dyer eine Haltung einnehmen, die die Formulierung „Gespräch mit der Erde“ in Bezug auf physiogeographisches Tun zwar in einer bestimmten Hinsicht für halbwegs tolerabel oder nachvollziehbar befindet, in anderer, grundsätzlicherer Hinsicht jedoch für unangemessen. So halten die Autoren die Formulierung dann für halbwegs tolerabel oder nachvollziehbar, wenn sie dazu dienen soll, sich von einem positivistisch oder naiv empiristisch geprägten Naturwissenschaftsverständnis abzugrenzen und auf den eben auch interpretativen Charakter des physisch-geographischen Tuns hinzuweisen. „Gesprächsähnlich“ finden sie dieses Tun dann insofern, als ein interpretatives Tun immer auch ein zirkuläres Moment besitzt, weil man seine eigenen Vorurteile oder Herangehensweisen dabei in Frage stellen muss. Für unangemessen wird die Formulierung jedoch deswegen befunden, weil - wie Demeritt und Dyer natürlich nicht $\mathrm{zu}$ Unrecht herausstellen - es etwas anderes sei, ob man es in seiner Forschung mit Menschen zu tun habe (bzw. mit sozialen oder kulturellen Phänomenen) oder eben mit nicht-menschlichen Dingen, die zwar interpretiert werden können, aber selbst nicht interpretieren. Die Autoren verweisen hier auf das bekannte Argument, dass die Sozialwissenschaften und somit auch die Humangeographie auf doppelte, nicht bloß auf einfache Weise hermeneutisch seien - und nur im Falle einer doppelten Hermeneutik sei es wirklich angemessen, von einem Gespräch zu sprechen. In diesem Zusammenhang wird nicht nur die Auffassung vertreten, dass die Gegenstände einer naturwissenschaftlichen Forschung keiner ,dichten Beschreibung“ bedürften im Sinne der Geertzschen ,thick description“, die z.B. nötig wäre, um ein ungewolltes Zucken eines Augenlids von einem kulturell bedeutungsvollen Zuzwinkern unterscheiden zu können. Zudem pointieren Demeritt und Dyer ihre Sichtweise, indem sie in ihrem Text einen fiktiven Vertreter der klassischen Hermeneutik auftreten lassen, der gegen eine Inanspruchnahme der hermeneutischen Idee des Gesprächs durch Naturwissenschaftler das Argument einbringt, dass ,rocks are not subjects, but mere objects - meaningless, inert and indifferent to the categories with which we represent them. As such they cannot speak or respond consciously to us so that truly intersubjective, hermeneutical dialogue is neither possible nor necessary“ (Demeritt und Dyer, 2002:236).

Dies genau ist der Punkt, an dem hier angesetzt und die Ausführungen von Demeritt und Dyer ergänzt werden sollen. So wird im Folgenden darzulegen versucht, dass es in der Physischen Geographie eine hermeneutische Dimension gibt, die nicht nur die Physische Geographie ebenfalls in gewisser Weise „doppelt hermeneutisch“ bzw. ,im Gespräch“ sein lässt, da sie jeglicher Interpretation von Beobachtetem 
vorausliegt, sondern die zudem und zugleich für die Existenz des Fachs sehr bedeutsam ist, weil sie so etwas wie seinen Kern, oder seine Grundprobleme, betrifft.

Den Bezug zu geographischen Grundproblemen herauszustellen ist auch in folgenden Hinsichten wichtig:

Erstens, um das rechte Verhältnis der folgenden Überlegungen zu der Philosophie Heideggers und Gadamers verstehen zu können, durch die sie inspiriert wurden wie auch andere, gewissermaßen vorarbeitende Aufsätze der Autorin (vgl. u.a. Zahnen, 2005, 2006, 2007, 2008a und 2008b). ${ }^{2}$ So wäre es ein Missverständnis anzunehmen, dass es einen entsprechenden Ansatz dieser Philosophien gäbe, der hier einfach hätte Anwendung finden können. Da die hermeneutische Phänomenologie bzw. hermeneutische Philosophie kein vorliegender „Ansatz“, keine bereitstehende Methode ist oder sein kann, sondern vielmehr einer nur durch eigene Einsichten zu gewinnenden Haltung entspricht, ist es für die folgenden Ausführungen wesentlich und im Sinne der hermeneutischen Phänomenologie bzw. Philosophie, dass sie aus der Geographie selbst heraus entwickelt wurden - oder genauer gesagt: auf der Basis von Erfahrungen und Problemen, die in dem Fach Geographie als solches auftauchen. Erst und nur daraus, d.h. aus einer eigens geund erlebten Kontexualität der Geographie heraus, vermag sich auch die Tragweite der Darlegungen zu ergeben - für das Fach Geographie wie über dieses hinaus.

Dessen gilt es - zweitens - auch eingedenk zu sein, wenn man sich im Zuge der folgenden Darlegungen verschiedentlich an Arbeiten von Latour (z.B. 1987) oder Pickering (z.B. 1995) - die ja keine Geographen sind - erinnert fühlen mag. ${ }^{3}$ Beweggrund und Potenzial des vorliegenden Aufsatzes, der völlig unabhängig von diesen Arbeiten entstand, lassen sich nicht auf scheinbar ähnliche Aussagen dieser Autoren reduzieren. Denn auch wenn die genannten Autoren - wie ich im Zuge des Aufsatzes ein in gewisser Weise wechselseitiges Verhältnis zwischen „Subjekt" bzw. Mensch einerseits und „Objekt“ bzw. Natur oder Materialität andererseits thematisieren mögen, so ist das Entscheidende des vorliegenden Aufsatzes, dass aufgezeigt wird, dass und wie sich Einsichten bezüglich derartiger

\footnotetext{
${ }^{2}$ Zur Rezeption hermeneutisch-phänomenologischen Denkens in den Naturwissenschaften, allerdings nicht erdbezogenen Naturwissenschaften, vgl. auch Kockelmans und Kisiel (1970), Heelan (1983, 1994, 1998 und 2002), Crease (1993 und 1997), Kisiel (1997), Kockelmans (1997) und Fehér et al. (1999). Dazu, dass durch den Einbezug bzw. die Explikation einer solchen hermeneutisch-phänomenologischen Dimension auch in den Naturwissenschaften eine Art „doppelte Hermeneutik“ zum Tragen kommt, vgl. u.a. Ginev (1997, 1999 und 2002) und Eger (1999).

${ }^{3}$ In den genannten Arbeiten, die Demeritt und Dyer in ihrer Einleitung (S. 229) übrigens selbst kurz erwähnen, werden Forschungspraktiken als eine dialektisch vermittelte Bewegung beschrieben, in der Forscher und Materialitäten gleichermaßen eine Rolle spielen.
}

Wechselverhältnisse aus der Physischen Geographie selbst heraus, als eigens gelebte Erfahrung, gewinnen lassen, und zwar auf eine Weise, die das physisch-geographische Selbstverständnis unumkehrbar zu verändern vermag. Was dies bedeutet, wird im Verlauf des Aufsatzes noch deutlicher werden.

\section{Die Frage nach den Bedingungen}

Dass es Erfahrungen gibt, die nicht von außen als kontextspezifisch zu analysieren sind, sondern eine eigens zu lebende und immer wieder neu zu erlebende Kontextualität überhaupt erst aufspannen und die eigene Situation somit auf irreversible Weise verändern, gilt es also in Bezug auf physisch-geographisches Tun zu verstehen. Auf dem Weg dahin werde ich den Begriff der Physischen Geographie und den der erdbezogenen Naturwissenschaften bzw. der Geowissenschaften zunächst quasi synonym verwenden auch wenn, wie bereits gesagt, im Verlauf des Aufsatzes eine Art brachliegender physisch-geographischer Kern eruiert werden soll, durch den sich das Fach von seinen sogenannten Nachbarwissenschaften zu unterscheiden vermag. Mit dieser anfänglichen Gleichsetzung soll - gerade wegen der Frage nach einem physisch-geographischen Kern - dem problematischen Umstand Rechnung getragen werden, dass das Selbstverständnis der Physischen Geographie derzeit so stark an dem Vorbild der verschiedenen erdbezogenen Naturbzw. Geowissenschaften orientiert ist, dass - zumindest auf den ersten Blick - ein wesentlicher Unterschied hinsichtlich der Fragestellungen und Vorgehensweisen nicht mehr zu existieren scheint. So betreiben sowohl Physische Geographen als auch Wissenschaftler der erdbezogenen Naturwissenschaften z.B. einerseits Studien, die in Anlehnung an die etwas überholte Windelbandsche Unterscheidung häufig noch als ,nomothetisch“ deklariert werden, und andererseits Studien, die man - „trotz“ ihres naturwissenschaftlichen Anspruchs - als ,idiographische“ Studien bezeichnet: ${ }^{4}$ Unter die erstgenannten Studien fallen all jene Bemühungen, die - meist aufgrund von Feldexperimenten oder Laborstudien - zu Gesetzen, Theorien, Modellen oder Repräsentationen über Ursache-Wirkungs- oder funktionale Wirkungsbeziehungen führen. Als „,idiographische“ Studien gelten in dem genannten naturwissenschaftlichen Kontext diejenigen, die, wie z.B. die Studien der historischgenetischen Geomorphologie, eine „Erklärung“ (besser: Interpretation) für die Genese bestimmter Erscheinungen in einem konkreten raumzeitlichen Kontext suchen, dabei aber die „nomothetisch“ erstellten Gesetze, Theorien, Modelle oder Repräsentationen anwenden, wenn auch häufig in miteinander verschachtelter Weise. Als „dritte“ Gruppe von Studien wären die Anwendungen komplexitätstheoretischer

\footnotetext{
${ }^{4}$ Vgl. dazu z.B. die Unterscheidung zwischen einer nomothetischen und einer idiographischen Geomorphologie in Ahnert (1996:17).
} 
Ansätze zu nennen, die etwa Chaos-Effekte und Instabilitäten in den Vordergrund rücken und mittlerweile sowohl in den erdbezogenen Naturwissenschaften als auch in der Physischen Geographie vorzufinden sind. Während ich mich zur Rolle solcher mathematischen komplexitätstheoretischen Ansätze an anderer Stelle bereits geäußert habe, ${ }^{5}$ soll hier anhand der oben skizzierten ,nomothetischen“ sowie ,idiographischen“ Studien der erdbezogenen Naturwissenschaften bzw. der Physischen Geographie eine grundlegende Frage aufgeworfen werden: die Frage nach den Bedingungen, unter denen die ,nomothetisch“ erstellten Gesetze, Theorien, Repräsentationen oder Modelle überhaupt sinnvolle Darstellungen abgeben und unter denen sie somit - in „idiographischen“ Bemühungen - auch sinnvoll anzuwenden sind. Diese Bedingungen sind nämlich aus den Theorien oder Modellen selbst nicht ableitbar.

Dass die Bedingungen irgendwie beachtet werden müssen, mag auf die Schnelle erst mal trivial klingen. Es ist aber alles andere als das. Denn die Frage ist, wie man dies versteht. Eine in sozialwissenschaftlichen Kontexten vermutlich häufig anzutreffende Argumentationsweise wäre, dass es eine Frage der Nützlichkeit oder Viabilität, der jeweiligen Mode oder auch der Macht ist, welche darüber entscheidet, welche Theorien oder Modelle überhaupt als sinnvolle Darstellungen oder auch als anzuwendende Theorien oder Modelle erscheinen. Und natürlich spielen derartige Momente oft genug auch eine Rolle. Ich bin jedoch der Meinung, dass die Wissenschaften zu einer Karikatur ihrer selbst verkommen würden, wenn allein diese gerade genannten Momente entscheidend wären. Es ließe sich auch nicht erklären, warum sich - wofür die wissenschaftshistorische Forschung eine Vielzahl von Beispielen liefern könnte - einzelne Wissenschaftler manchmal jahrelang mit einem wissenschaftlichen Problem herumschlagen, obwohl ihnen in keinster Weise klar ist, was herauskommen wird, und obwohl es ihrer Karriere oft mehr schadet als nützt. In diesen Situationen, die ja in der Wissenschaftsgeschichte immer wieder auch zu großen wissenschaftlichen Entdeckungen geführt haben, kommt offenbar etwas zum Tragen, das für einen Wissenschaftler eigentlich allein aus dem Vollzug des eigenen wissenschaftlichen Tuns heraus Geltungskraft entwickelt, das in gewisser Weise über die Grenzen des jeweiligen historischen Kontexts bereits hinausweist und in seiner tragenden Prozessualität für einen Beobachter zweiter Ordnung bzw. in einer sozialwissenschaftlichen Naturwissenschaftsforschung gar nicht in den Blick geraten kann. Ich möchte es, auch aus einem Grund, der später noch deutlicher werden sollte, den tragenden Anspruch der Sache nennen. ${ }^{6}$

\footnotetext{
${ }^{5}$ In einem Vortrag, den ich unter dem Titel „Zum paradigmatischen Potenzial geomorphologischer Begrifflichkeiten" am 20.9.2009 auf dem Deutschen Geographentag in Wien gehalten habe.

${ }^{6}$ Wie sich an dieser Stelle wie auch in den folgenden Ausführungen zeigt, ist das Zustandekommen der Formulierung
}

\section{Evolutives Tun}

Um darlegen zu können, inwiefern ein tragender Anspruch der Sache auch in den erdbezogenen Naturwissenschaften bzw. der Physischen Geographie eine Rolle spielen kann und muss, und zwar auch in der ,alltäglichen“ wissenschaftlichen Praxis, nicht nur in absoluten Ausnahmesituationen, möchte ich zunächst auf eine Form naturwissenschaftlichen Tuns aufmerksam machen, die hier als ,evolutives“ Tun bezeichnet sei. Für eine ausführliche Darlegung des Hintergrunds meiner Formulierung des Begriffs des „Evolutiven“, der sich an den ja sowohl in der Physischen Geographie (z.B. Thornes, 1983) als auch - wie z.B. bei Rheinberger (2007:89ff.) nachzulesen ist - in der Wissenschaftsforschung nicht ungebräuchlichen Begriff des Evolutionären anlehnt, zugleich aber auch davon abgrenzt, fehlt hier der Platz. Wichtig ist allerdings, dass die Existenz und grundlegende Bedeutung dieses evolutiven Tuns mir interessanterweise im Zuge von Überlegungen bewusst und verständlich geworden ist, in denen ich mich der Frage einer möglichen Anschluss- oder Begegnungsmöglichkeit und somit auch einer möglichen Kommensurabilität zwischen den mathematischen Komplexitätstheorien, die derzeit als ,neues Paradigma“ der Physischen Geographie gehandelt werden, und den traditionellen ,nomothetischen“ bzw. ,idiographischen“ Ansätzen der Physischen Geographie gewidmet habe. Dieser Umstand kann uns nämlich als Hinweis darauf gelten, dass das Phänomen eines tragenden Anspruchs der zu untersuchenden Sache auch die Frage des Verhältnisses verschiedener wissenschaftlicher „Paradigmen“ zueinander betrifft, ja diese überhaupt erst in ein Verhältnis zueinander zu rücken vermag, d.h. eine Art Basis für deren mögliches Verhältnis schafft.

Wesentlicher Punkt des ,evolutiven“ - nicht als ,evolutionär" misszuverstehenden - naturwissenschaftlichen Tuns ist, dass es sich um ein Tun handelt, in dem das, was Gegenstand der eigenen Bemühungen ist, nicht schon vorausgesetzt wird - wie der ,rock“ in dem weiter oben (Abschnitt 2) zitierten Argument eines fiktiven Vertreters der

„tragender Anspruch der Sache“ nicht im Sinne einer Übernahme von definierten Fachtermini - etwa einer ganz bestimmten Philosophie - zu verstehen. Zwar lässt sich schon sagen, dass z.B. die hier zur Sprache gebrachte "Sache“ dem Begriff der Sache in Gadamers Wahrheit und Methode (1999c) näher steht als etwa demjenigen einer bewusstseinsorientierten, thematischen Phänomenologie Husserls. Trotzdem ist es wichtig zu sehen und ganz im Sinne der Philosophie Heideggers und Gadamers -, dass sich die Formulierung aus dem eigenen Vollzug des Gedankengangs des vorliegenden Aufsatzes im Wechselspiel mit den entbergenden Möglichkeiten einer lebendigen, gelebten Sprache, ,in der wir immer schon sind“ herausgebildet hat und nicht einer aus allen lebendigen Bezügen abgehobenen definierbaren Begriffssprache entspricht, die man einfach so übernehmen könnte (zum hermeneutischen Verständnis von Sprache, gerade in Bezug auf den Begriff der Geographie, vgl. auch Zahnen, 2007). 
klassischen Hermeneutik im Text von Demeritt und Dyer. Vielmehr bildet es sich überhaupt erst einmal heraus. Das evolutive Tun kann daher in einem ersten Schritt als ein Tun verstanden werden, das sich in der Entstehungsphase bzw. in der Bildungs- und Umbildungsphase von Gebilden bewegt und somit auch deren Bedingtheit in Erfahrung bringt.

In den experimentellen Laborwissenschaften kann man sich ein solches Tun so vorstellen, dass es gerade anders vorgeht als die klassischen Laborexperimente eines mechanistischen Weltmodells. Die Randbedingungen werden nicht möglichst konstant gehalten, um die Auswirkungen der Veränderungen eines einzelnen Parameters messen zu können, sondern die Randbedingungen werden gerade variiert, um überhaupt erst einmal in Erfahrung zu bringen, welche Bedingungen oder Parameter für ein Phänomen oder Gebilde überhaupt wesentlich sein könnten bzw. wie sich ein Gebilde unter den wandelnden Umständen verhält. Man versucht herauszufinden, wann es in Erscheinung tritt bzw. verschwindet, ob es sich bloß quantitativ verändert oder ob es zu einem qualitativen Wandel kommt, so dass man es eigentlich mit einem anderen Phänomen zu tun hat. Als konkretes Ergebnis eines solchen Tuns könnte man z.B. herausbekommen, dass ein bestimmter Prozess bei bestimmten Werten eines Parameters $\mathrm{X}$ auftaucht und zunächst nur von diesem abhängt, dann aber bei größeren Werten von $\mathrm{X}$ aber zusätzlich von einem Parameter $\mathrm{Y}$ bestimmt wird und sich schließlich bei noch größeren $\mathrm{X}$ wieder auflöst, während nun ein anderer Prozess auftaucht. Des Weiteren wäre z.B. denkbar, dass man einen Hysterese-Effekt, also eine gewisse Pfadabhängigkeit in Erfahrung bringt, so dass es nicht egal ist, wie bzw. von wo her kommend man die Bedingungen variiert, ob man also z.B. bei kleinen Werten beginnt und zu größeren Werten fortschreitet oder umgekehrt.

Diese Darstellung möglicher Ergebnisse eines evolutiven Vorgehens in den experimentellen Naturwissenschaften darf man aber nun nicht missverstehen. Entscheidend an einer solchen Erfahrung von Bildungen und Umbildungen von Gebilden ist nämlich weniger das, was späterhin als Beziehung zwischen konkreten Bedingungen und konkreten, beobachtbaren Wirkungen irgendwie festgehalten und quasi als Repräsentation eines durch Naturfaktoren bestimmten Zusammenhangs weitergegeben werden kann. Wesentlicher Zug des evolutiven Tuns ist nicht, dass dabei ein Wissen entstehen würde, das sich allein auf eine Welt objektartiger Gebilde bezöge und in Form abbildartiger Repräsentationen, Theorien oder Modelle von bestimmten Naturgebilden dargestellt werden könnte - ein Eindruck, der in einer rein naturwissenschaftlichen Lesart schnell entstehen könnte. Genauso wenig geht es aber darum, allein den Part des Wissenschaftlers herauszustellen, der in seinem Experiment die Umstände aktiv variiert und so bestimmte Ergebnisse produziert bzw., wie Sozialwissenschaftler zu sagen geneigt wären: konstruiert. Das Entscheidende an dem evolutiven Tun, das ich hier herausstellen möchte, ist vielmehr, dass es in seinem evolutiven Vollzug zu einem ebenfalls als evolutiv zu verstehenden Wissen führt, dessen Eigenart darin besteht, Einsichten zu entsprechen, welche das Verhalten der sogenannten Objekte und die Verhaltensweise der sogenannten Subjektseite gleichermaßen betreffen. Denn wollte man die Einsichten formulieren - was im Allgemeinen nicht geschieht, weil sie sich meist als tacit knowledge (Polanyi) manifestieren - dann würden sie etwa so lauten: „Hier bin ich zu weit gegangen, hier habe ich die Umstände zu weit variiert, das Gebilde ist verschwunden oder qualitativ ein anderes geworden". Genauso aber auch: „Hier kann ich oder muss ich noch ein Stück weiter gehen, bis es zu einer qualitativen Veränderung kommt“. Diese Sätze soll hier zunächst zwei wesentliche Aspekte deutlich werden lassen: Zum einen, dass es sich bei einem evolutiven Wissen um ein Wissen handelt, das sich nur aus dem eigenen Tun oder Vollzug heraus bilden kann eben dadurch, dass man sich selbst in der Bildungs- und Umbildungsphase von Gebilden bewegt. Zum anderen, dass sich bei einem solchen sich herausbildenden Wissen gar nicht sagen lässt, ob es von uns stammt, oder von den Dingen bzw. der sogenannten materiellen Dimension. Es ist jenseits einer Subjekt-Objekt-Spaltung zu verorten. Das evolutive, sich bildende, umbildende und in diesem Sinne ein bestimmtes Verhalten aufweisende Naturgebilde ermöglicht die evolutive, sich bildende und umbildende Verhaltensweise des Wissenschaftlers und umgekehrt.

\section{Evolutives Tun im Gelände}

Löst man sich aber nun von dem Beispiel experimenteller Naturwissenschaften und überträgt den letzten Satz auf die im Gelände tätigen erdbezogenen Naturwissenschaften, z.B. die Geomorphologie, dann scheint man zunächst auf ein Problem zu stoßen. Es scheint nicht angemessen zu sein, davon zu sprechen, dass die sich bildende und umbildende Verhaltensweise des Wissenschaftlers auch irgendwie die Bildung eines Naturgebildes ermöglichen kann, und ebensowenig, dass sich ein Wissenschaftler in der Bildungs- und Umbildungsphase von Naturgebilden bewegt - schließlich liegt diese doch, z.B. im Fall der Geomorphologie, zu weiten Teilen schon lange zurück in einer Vergangenheit geologischen Ausmaßes, in der es u.U. noch gar keine Menschen gab. Doch das ist zu kurz und zu konkret gedacht: Nicht nur, weil dann eine Trennung zwischen dem Bildungsprozess eines Naturgebildes und dem des Wissenschaftlers vorgenommen wird, die es in einem evolutiven Tun, in dem ein Naturgebilde in seiner eigenen Qualität überhaupt erst einmal in Erfahrung gebracht wird, gar nicht gibt. Auch, weil ein wesentliches Charakteristikum dieser im Gelände oder Feld tätigen erdbezogenen Wissenschaften ist, dass sie sich auf noch radikalere Weise als die im Labor evolutiv vorgehenden experimentellen Naturwissenschaften in der Bildungs- und Umbildungsphase von Naturgebilden 
bewegen. Radikaler deswegen, weil ihnen, anders als den Laborwissenschaften, die Möglichkeit fehlt, zumindest noch die Ausgangsbedingungen ,herzustellen“, unter deren Voraussetzung ein Hervortretenlassen und zugleich Auffindenlassen von Naturerscheinungen durch ein Bewegen in der übergänglichen Bildungs- und Umbildungsphase in Gang gebracht werden muss. Bei den im Gelände tätigen erdbezogenen Naturwissenschaften gibt es eine derartige Schaffung von Ausgangsbedingungen nicht, da wir uns in diesen immer schon bewegen. ${ }^{7}$

So geht es in diesen erdbezogenen Naturwissenschaften gerade eben nicht um den isoliert und statisch vorliegenden „rock“, der bloßes, vorausgesetztes Objekt ist und erst in einem zweiten Schritt unseren Interpretationen unterliegt - um nochmals auf das Argument zurückzukommen, das Demeritt und Dyer einen fiktiven Vertreter der klassischen Hermeneutik haben anführen lassen, um eine doppelte Hermeneutik in den Naturwissenschaften in Abrede zu stellen. Sondern es geht eigentlich immer um in sich vielschichtige Gebilde, die wir zwar in unseren Repräsentationen in eine Vielzahl von Gegenständen differenter räumlicher und zeitlicher Skala aufzuspalten geneigt sind - vom Regentropfeneindruck und Spülrillen bis hin zu Gebirgszügen und Kontinentalschilden. Und tatsächlich fehlen in kaum einem geographischen Lehrbuch Abbildungen dieser Art (vgl. z.B. Ahnert, 1996:7). Aber die Gebilde begegnen uns ja im Gelände nie so aufgespalten, und vor allem nicht als geschlossene, alle einzelnen Elemente klar und deutlich umfassende statische Ganzheit, sondern im Prozess der Geländeerkundung, im Vollzug einer physischen Bewegung durch das Gelände, stets auf spezifische Weise ineinander verschachtelt und übergänglich - eben als sich wandelnde Gebilde. Daher bewegen wir uns auch bei der Geländeerkundung in der übergänglichen Bildungs- und Umbildungsphase von Naturgebilden und bilden uns - das ist entscheidend - dabei selbst um bzw. weiter.

Die dadurch gewonnene und überhaupt erst ermöglichte Geländeerfahrung - der Erfahrungsbegriff kommt hier, ganz in hermeneutischer Tradition, in seinem Doppelsinn zum Tragen - geht ebenso aus Einsichten hervor, die unser Verhalten wie auch das Verhalten bzw. die Verhältnisse des Geländes gleichermaßen betreffen. Diese Gelände-Einsichten ließen sich ebenso durch die Sätze charakterisieren, die oben in Bezug auf das evolutive Tun der Laborwissenschaften angeführt wurden: ,Hier bin ich zu weit gegangen, hier habe ich die Umstände zu weit variiert, das Gebilde ist verschwunden oder qualitativ ein anderes geworden“. Oder aber auch: „Hier kann ich oder muss ich noch ein Stück weitergehen, die Umstände noch weiter

\footnotetext{
${ }^{7}$ Wobei gesagt werden muss, dass das besagte „Herstellen“ einer experimentellen Ausgangssituation natürlich auch nicht ohne geschichtlichen Hintergrund ist. Der angeführte Unterschied zu den im Gelände tätigen erdbezogenen Naturwissenschaften bleibt davon aber unberührt.
}

variieren, bis sich eine qualitativ wesentliche Veränderung ergibt oder ergeben könnte.“

Doch hier sei nun auf einen weiteren Aspekt hingewiesen, der sich, wenn man mit der deutschen Sprache vertraut ist, aus den gerade angeführten umschreibenden Sätzen herauslesen lässt: Dass das entscheidende, qualitätsbestimmende Wissen, das jenseits oder diesseits einer Subjekt-ObjektSpaltung liegt, eine Art Taktgefühl ist - etwas, das ich „physisch-geographischen Takt" nennen möchte.

\section{Takt - und Geländeerkundung}

Nun bin ich mir bewusst, dass den Begriff des Taktes im Zuge der erdbezogenen Naturwissenschaften bzw. der Geographie zu verwenden nicht neu ist - er findet sich in vielen entsprechenden Schriften bereits des 19. Jahrhunderts. Trotzdem gilt es zu verstehen, dass sich der Verweis auf die Dimension des Taktes nicht aus mehr oder weniger gedankenloser Anlehnung an derartige Vorgänger, sondern aus den obigen Darlegungen zu einem evolutiven, sich in der Bildungs- und Umbildungsphase bewegenden Tun während der Geländeerkundung ergibt - und was dies bedeutet. Denn vor diesem Hintergrund ist es weniger bedeutsam, dass und von wem der Begriff des Taktes wann oder wo bereits verwendet worden ist, als vielmehr die Frage, ob seiner Verwendung jeweils der Beweggrund zugrunde liegt, das eigene wissenschaftliche Tun weiterzubringen und besser verstehen zu lernen. ${ }^{8}$

Takt ist ja nichts, das man ein für allemal hat, sondern etwas, das nur ist, indem es sich im Vollzug, in der Weise, wie

\footnotetext{
${ }^{8}$ Dies muss sicherlich nicht immer der Fall sein. So möchte ich nicht ausschließen, dass im Verlauf der geographischen Fachgeschichte der Begriff des Taktes auch - ohne aus dem genannten Beweggrund hervorgegangen $\mathrm{zu}$ sein - bloß übernommen und eingesetzt wurde, um damit z.B. methodische oder theoretische Mängel, etwa hinsichtlich der Abgrenzung von Räumen, zu kaschieren oder um sich durch Berufung auf einen solchen geographischen Takt - als einer dem Fach eigenen „Methode“ in Zeiten eines fraglich gewordenen Selbstverständnisses schlechterdings legitimiert zu fühlen. Dies würde die äußerst kritische Haltung gegenüber einer Berufung auf den geographischen Takt rechtfertigen, wie sie z.B. auch bei Lippuner (2005:14) zu finden ist, der den geographischen Takt als ,ominös“ bezeichnet. Allerdings mag ich genausowenig ausschließen, dass es Geographen oder erdbezogene Naturwissenschaftler gab, die - ähnlich wie ich in diesem Aufsatz - den Begriff des Taktes tatsächlich deswegen gewählt haben, um damit etwas zum Ausdruck zu bringen zu versuchen, das sie als eine wirksame Dimension der Praxis ihres geographischen Tuns selbst kennen gelernt haben und darstellungswert oder gar -notwendig finden. Dies ist eine eigene Untersuchung wert. Die Frage hinsichtlich der Verwendung des Begriffs des Taktes ist dabei letztlich, ob Sprache als lebendiges Geschehen im Sinne eines Primats des Bildes - statt der Schrift - gelebt wird oder nicht. Zu dieser Frage, die den Kern der Kontroverse zwischen Gadamer und Derrida betrifft, in Bezug auf geographisches Tun vgl. Zahnen (2007).
} 
man sich in einer konkreten Situation verhält, zeigt. Es geht um einen Formfindungsprozess, der für einen selbst einem Gegenüber in seiner jeweiligen Situation angemessenen ist. Takt verweist uns auch durch seine Verwandtschaft zum Begriff der Taktilität ja auf so etwas wie ein Fingerspitzengefühl und somit auch eine leibliche Dimension. Beide Aspekte vereinigen sich nun in der Geländeerkundung: Die Weise, wie sich jemand im Zuge der Geländeerkundung verhält, dann, wenn er sich im zweifachen Sinne in der Bildungs- und Umbildungsphase des Geländes bzw. von immer irgendwie einzigartigen, in sich vielschichtigen Naturgebilden bewegt, ist ein Prozess der Formsuche und Formfindung, der zu einer Herausbildung eines sich leiblich manifestierenden Gespürs, einer leiblichen Erfahrenheit führt, zugleich aber auch von einer solchen leiblichen Erfahrenheit geleitet wird. Der physisch-geographische Takt kommt daher gerade in dem formsuchenden und -findenden Verhalten zum Ausdruck, das sich nicht formalisieren lässt, für das es keine methodische Anleitung, kein Skript, keinen immergültigen, bereits festgelegten oder vorauszusetzenden Katalog gibt, und das es gerade deswegen braucht, weil man es im Gelände mit immer wieder anderen, sich ständig wandelnden übergänglichen und in sich vielschichtigen Umständen bzw. Gebilden zu tun hat. Er zeigt sich in einem unausdrücklichen - selbstverständlich nie absolut sicheren - Wissen oder Gespür darum, ob bestimmte Übergänge bzw. Aspekte eines Geländes übergangen werden können oder nicht, ob man noch weiter gehen oder nochmals zurückgehen oder das Gelände bzw. Gebilde nochmals auf andere Weise erfahren oder betrachten muss oder nicht, ob man also sich - und in gewisser Weise ja auch dem Anspruch des Geländes bzw. der sogenannten materiellen Dimension - noch mehr Bildungsbzw. Umbildungsmöglichkeiten ermöglichen muss oder nicht. Mit anderen Worten: Der physisch-geographische Takt zeigt sich in einem Gespür dafür, ob man ausreichend ins Bild gekommen ist, ob die Erfahrung eines Gebildes bzw. Geländes - trotz einer prinzipiellen Unabschließbarkeit - als vorläufig abgerundet und in sich stimmig gelten darf - oder ob man noch länger in der Bildungs- und Umbildungsphase verweilen sollte. Und er ist nichtzuletzt dadurch motiviert, dass der, der das Gelände erkundet, erst einmal herausfinden will, was am jeweiligen Ort und zur jeweiligen Zeit - wie man umgangssprachlich so trefflich sagt - ,überhaupt Sache ist". Das heißt: Auch der taktvolle Formfindungsprozess der Geländeerkundung folgt einem tragenden Anspruch der Sache.

Darin gleicht die Situation der Geländeerkundung den oben bereits erwähnten Situationen, die zu großen wissenschaftlichen Entdeckungen, zu völlig neuen Sichtweisen geführt haben, d.h. den Situationen, in denen etwas zum Tragen kommt, das für einen Wissenschaftler eigentlich allein aus dem Vollzug des eigenen wissenschaftlichen Tuns heraus Geltungskraft entwickelt und in seiner tragenden, über die Grenzen des jeweiligen historischen Kontexts hinausweisenden Prozessualität für außenstehende
Beobachter eines naturwissenschaftlichen Tuns gar nicht erfahrbar sein kann. Daher ist die leibliche Erfahrenheit, die in den erdbezogenen Naturwissenschaften zum Tragen kommt, auch nicht derart zu verstehen, dass sie bloß einem durch Habitualisierungen ermöglichten Wiederfinden oder $R e$-produzieren von Erscheinungen dienen würde - etwa derart, wie es in den Laborwissenschaften eines nicht nur metaphorisch zu verstehenden „Händchens“ oder Fingerspitzengefühls bedarf, um Proben derart zu präparieren, Kulturen derart anzulegen oder Geräte derart justieren zu können, dass bestimmte Phänomene wiederholt produziert werden können - auch wenn diese Dimension zu begreifen von großer Wichtigkeit ist. Ganz abgesehen davon, dass die Naturerscheinungen der Erde - neben der Tatsache, dass sie sich eingebettet in Dimensionen abspielen, die sie jenseits unserer „Verfügbarkeit“ hält - einem ständigen räumlichen und zeitlichen Wandel unterliegen und sich daher gar nicht wiederholen ließen: Nicht die sich in Wiederholungen niederschlagende, sondern die öffnende, ins Bild kommen lassende, das heißt welterschließende Dimension der leiblichen Erfahrenheit eines Wissenschaftlers ist das, was es eigentlich $\mathrm{zu}$ verstehen gilt. So zeigt sich der erfahrene und d.h. taktvolle Wissenschaftler im Gelände auch nicht darin, dass er genau wüsste, was vorliegt, oder immer schon wüsste, was er vorfinden wird. Er zeigt sich vielmehr darin, dass er es ist, der eine Nase für das Übergangene und Übersehene hat, dafür, wo man wie vielleicht auch nochmals hingehen, was man wie vielleicht nochmals anschauen, wie das in sich vielschichtige und übergängliche Naturgebilde noch weiter erkundet werden müsste usw. Ich möchte es so auf den Punkt bringen: Der taktvolle Wissenschaftler hat ein Gespür für das Vergessene und den Anspruch, diesem Anspruch der Sache zu folgen. Das Entscheidende dieses sich leiblich manifestierenden Anspruchs der Sache - und auch des physisch-geographischen Takts - ist seine Offenheit auf etwas hin, das man nicht wirklich $\mathrm{zu}$ fassen vermag, auch wenn man dazu schon irgendwie - ahnend in Bezug steht. In diesem Sinne verstehe ich den Anspruch der Sache als tragend. Er trägt und führt den Wissenschaftler in bisher übergangene oder unverstandene Möglichkeiten des Geländes bzw. (Fach-)Gebiets, in dem er sich bewegt. Er lässt den Wissenschaftler auf neue Weise ins Bild kommen.

\section{Takt - als Grundbedingung der erdbezogenen Naturwissenschaften}

Ein solcher Takt, ein solcher tragender Anspruch ist freilich in jedem neuen Einzelfall, im jeweiligen Vollzug der auf Geländeerfahrenheit basierenden Geländeerfahrung, also durch die Begegnung mit der sogenannten materiellen Dimension in ihrer jeweils einzigartigen Übergänglichkeit immer wieder neu gefordert. Und dies gilt auch für die Durchführung der sogenannten „,nomothetischen“ bzw. der sogenannten ,idiographischen“ Studien der erdbezogenen 
Naturwissenschaften, wie sie heute auch Physische Geographen betreiben: In den ,nomothetischen“ Studien betrifft dies vor allem den Prozess der Auswahl eines Messstandortes für Feldexperimente, die ja im Allgemeinen der empirischen Erfassung eines ganz bestimmten Prozesses dienen sollen und somit eines entsprechend geeigneten und eingegrenzten Messstandortes bedürfen, was angesichts der Verschränkung von Prozessen unterschiedlicher räumlicher und zeitlicher Skala oft gar nicht so leicht zu bewerkstelligen ist. In den „,idiographischen“ Studien ergibt sich die Notwendigkeit im Zuge der spurenlesenden historisch-genetischen Interpretationen abduktiven Charakters, deren Charakteristikum es ja gerade ist, dass es immer mehrere Interpretationsmöglichkeiten gibt. In beiden Fällen muss sich der im Gelände tätige Wissenschaftler eigentlich ständig ,fragen“, womit er es eigentlich zu tun hat, ob noch damit, womit er es zu tun zu haben glaubte, oder nicht eventuell (auch) mit ganz anderen Verhältnissen oder Prozessen, was eine weitere bzw. revidierende Geländeerkundung erforderlich machen würde. Und auch wenn dieses Fragen natürlich meistens nicht in expliziter Form geschieht, sondern sich in der Weise zeigt, wie sich der Wissenschaftler in seinem Gelände verhält, so ist es doch gerade dieser von der Struktur her einem Fragen gleichende, sich von einem Gespür leiten lassende Prozess, der Studien der erdbezogenen Naturwissenschaften überhaupt qualitativ wertvoll werden lässt. Gespür, geographischer Takt, etwas, das man im Zuge der in der zweiten Hälfte des 20. Jahrhunderts vorangetriebenen Szientifizierung der Geographie in eins mit der traditionellen Landschaftsgeographie loswerden zu müssen gedachte, erweist sich somit - ganz gegenteilig - als eine Grundbedingung qualitativ ernstzunehmender Arbeiten in den erdbezogenen Naturwissenschaften. Müssen die im Gelände tätigen erdbezogenen Naturwissenschaftler - jenseits der Frage der Nützlichkeit und angesichts des sich stets wandelnden Charakters der Naturgebilde - soweit wie irgend möglich die Bedingungen berücksichtigen, unter denen die von ihnen erstellten Theorien, Modelle, Gesetze oder Repräsentationen sinnvolle Darstellungen abgeben oder sinnvoll anzuwenden sind - und dies müssen sie trotz und auch wegen aller historischen bzw. kulturoder sozialwissenschaftlich fassbaren Überprägungen der wissenschaftlichen Praktiken tun -, so tun sie dies, sofern nicht mathematische Wege gesucht werden, letztlich immer auch stillschweigend und unausdrücklich im Vollzug ihres physisch-geographischen Takts auf der Basis und in Form einer anspruchsvollen Geländeerkundung.

\section{8 „Doppelte Hermeneutik“}

Genau dies heißt letztlich aber nichts anderes, als dass jede sogenannte ,idiographische“ wie auch jede sogenannte ,nomothetische" Studie der erdbezogenen Naturwissenschaften nur dadurch qualitativen Wert erlangt, dass sie aus einem Verhalten hervorgeht, das öffnend oder „welterschließend“ in dem Sinne ist, dass ein Naturgebilde - bzw. das Gelände und Gebiet, in dem man sich bewegt - in seiner eigenen Qualität überhaupt erst erfahrbar wird. Auf dieser grundlegenden Ebene wird der Gegenstandsbezug derartiger Studien überhaupt erst ermöglicht. Unter Berücksichtigung dieser Ebene, die im Heideggerschen Sinne als hermeneutisch $\mathrm{zu}$ verstehen ist und allen Interpretationen und Re-Interpretationen eines wissenschaftlichen Gegenstands vorausgeht - auch jeder methodologischen oder epistemologischen Reflexion -, gibt es dann aber eben auch in den erdbezogenen Naturwissenschaften eine gewisse Art ,doppelter Hermeneutik“. Zwar würde ich es weiterhin nicht für angemessen halten, das wechselseitige Verhältnis zwischen einem sich bildenden und umbildenden Wissenschaftler und einem sich bildenden und umbildenden Naturgebilde, aus dem sein Gegenstandsbezug hervorgeht, als eine Form der Intersubjektivität zu bezeichnen, wie sie in einem zwischenmenschlichen Gespräch zum Tragen kommt. Doch das im Vollzug der erdbezogenen Naturwissenschaften zum Tragen kommende wechselseitige Verhältnis ist wie Intersubjektivität - ebenso ein Drittes, das über die jeweiligen einzelnen Sphären der „Teilnehmer" dieses Geschehens hinausgeht. Das „Dritte“, das hier trägt und die Bildungs- und Umbildungsmöglichkeiten, d.h. ein immer nur vorläufiges Ins-Bild-kommen ermöglicht, ist eben die Sphäre des Anspruchs der Sache. Dieser Anspruch der Sache ist immer ein zweifacher: Er ist der Anspruch des eben anspruchsvollen - Wissenschaftlers wie der Anspruch des - eben anspruchsvollen - Geländes bzw. Naturgebildes gleichermaßen.

So sprechen die in sich vielschichtigen und übergänglichen Naturgebilde der Physischen Geographie natürlich nicht wie wir Menschen sprechen. Aber sie führen in dem Sinne eine Sprache, als sie in dem Prozess, in dem sie sich einem überhaupt erst herausbilden, im eben dargelegten zweifachen Sinne anspruchsvoll sind und somit immer auch schon über sich hinausweisen. So will ja auch Gadamer anhand des Phänomens des Gesprächs mehr zum Ausdruck bringen als einen ontischen Kommunikationsvorgang zwischen zwei Subjekten in ihrer jeweiligen Subjektivität, nämlich ein die Subjektivität des Subjekts überschreitendes, sich nie aus einer Sphäre des Unverstandenen völlig herauslösendes Seinsgeschehen. ${ }^{9}$ Derart ist auch Gadamers berühmt gewordener Satz „Sein, das verstanden werden kann, ist Sprache“ zu verstehen. ${ }^{10}$ Auch Dinge, auch

\footnotetext{
${ }^{9}$ Vgl. dazu z.B. Gadamer (1999b:335).

${ }^{10}$ Wie Gadamer selbst schreibt, vgl. Gadamer (1999b:334).
} 
Naturgebilde der Erde können in diesem Sinne Sprache sein, sofern sie medial wirken, also auf eine Weise, die uns in einen öffnenden Bezug zu der Sphäre eines Unverstandenen hält, d.h. uns ein Ins-Bild-kommen ermöglichen. ${ }^{11}$ Diese Sprache zu „können“ heißt mehr, als ein Vokabular zu beherrschen. Es macht im zweifachen Sinne anspruchsvolles naturwissenschaftliches Tun aus.

\section{Takt - und Geographizität}

Zu Beginn des vorliegenden Aufsatzes wurde gesagt, dass es so etwas wie einen brachliegenden, impliziten Kern Physischer Geographie gäbe, ohne den diese auch in ihrer derzeitigen, den erdbezogenen Naturwissenschaften angeglichenen Ausprägung nicht ernsthaft auskäme. Der darauf folgende, bisherige Gedankengang hat gezeigt, dass dieser Kern, dieser Grundzug, einen nur im eigenen Vollzug zum tragenden kommenden, die Leiblichkeit des Menschen einbeziehenden und mit einer „Sprache“ irdischer Naturgebilde resonierenden physisch-geographischen Takt betrifft, der ein Gelände bzw. irdische Naturgebilde in ihrer einzigartigen Qualität und Übergänglichkeit überhaupt erst und immer wieder neu erfahrbar werden lässt und somit auch entsprechende geographische bzw. naturwissenschaftliche Gegenstandsbezüge überhaupt erst und immer wieder neu ermöglicht. „Ernsthaft auskommen“ kann eine Physische Geographie derzeitiger Ausprägung - und somit auch jede entsprechend arbeitende erdbezogene Naturwissenschaft ohne diesen Kern zudem deswegen nicht, weil er, wie gezeigt wurde, ihre naturwissenschaftlichen Darstellungen für den Kundigen überhaupt erst sinnvoll bzw. ihre Arbeiten qualitativ wertvoll werden lässt. ${ }^{12}$ Inwiefern kann die Einsicht in derartige Grundzüge physisch-geographischen Tuns aber - wie eingangs ebenfalls gesagt wurde ein neues Selbstverständnis und insofern auch eine neue Aufgabe für die Physische Geographie bedeuten: eine Physische Geographie im starken Sinne, welche nicht mehr in dichotomer Entgegensetzung zur Humangeographie zu verstehen wäre?

Hier kann nun ein weiterer Aspekt des physischgeographischen Takts ins Spiel gebracht werden. Dabei spreche ich nun bewusst von einem physisch-geographischen Takt und nicht von einem Takt der erdbezogenen Naturwissenschaften, obwohl ja auch die Geologie, die Meteorologie usw. eines solchen Taktes bedürfen, um die Qualität ihrer Arbeiten zu gewährleisten. Somit wird im Folgenden die für

\footnotetext{
${ }^{11}$ Gadamer hat auf eine solche Möglichkeit einer Sprache der Dinge oder der Natur selbst hingewiesen, vgl. dazu Gadamer (1999c:478) sowie Gadamer (1999a). Dazu auch: Hogrebe (2004), wo sich auch der Bezug zwischen diesem Aspekt der Gadamerschen Philosophie und der Philosophie Heideggers andeutet.

${ }^{12}$ Wobei nochmals darauf hingewiesen sei, dass es auch - in diesem Aufsatz nicht weiter diskutierte - mathematische Wege gibt, zu ermitteln, ob bestimmte Darstellungen sinnvoll bzw. sinnvoll anzuwenden sind.
}

die Identität des Fachs Geographie problematische Gleichsetzung von „Physischer Geographie“ und „erdbezogenen Naturwissenschaften" wieder aufgehoben, die oben zunächst vorgenommen wurde, um dem Umstand Rechnung zu tragen, dass derzeit zwischen Studien, die Wissenschaftler der verschiedenen erdbezogenen Naturwissenschaften betreiben, und solchen, die von Physischen Geographen durchgeführt werden, zumindest auf den ersten Blick kein Unterschied mehr besteht.

Hintergrund für die nun nicht mehr außer Acht gelassene Unterscheidung ist die Überlegung, dass sich die Physische Geographie, anders als ihre sogenannten „Nachbar"wissenschaften wie die Bodenkunde, Geologie, Meteorologie usw., noch nicht auf ein bestimmtes Element bzw. einen bestimmten Aspekt der Naturerscheinungen der Erde eingeschossen oder begrenzt hat. Ihre Sache weist also in sich eine wesentlich vielschichtigere Übergänglichkeit auf. Sie bewegt sich in einer den verschiedenen $\mathrm{Ge}$ genständen der erdbezogenen Naturwissenschaften zugrundeliegenden, das heißt wesentlich weiteren Übergänglichkeit bzw. Bildungs- und Umbildungsphase von Naturgebilden. Ihr Anspruch trägt, tiefer gründend, notwendig weiter.

Ein weiterer wichtiger Punkt ist aber, dass das Problem des Taktes selbst wesentlich mit einem Darstellungsproblem verknüpft ist und uns somit auf das Graphische oder die Graphizität der Geographie zurückwirft. ${ }^{13}$ Die Verbindung zwischen dem Phänomen des Taktes und einer Darstellungsproblematik wird im Bereich des zwischenmenschlichen Umgangs ja dadurch deutlich, dass sich ein taktvolles Vorgehen gerade darin zeigt, dass man etwas ungesagt lässt oder übergeht. Man versucht, Taktlosigkeiten zu vermeiden, indem man nichts sagt, was nicht gesagt werden muss. Zugleich stellt man aber sein Taktgefühl gerade dann unter Beweis, wenn man jemanden auf etwas hinweisen muss, das ihm vielleicht auch unangenehm ist, aber sich von der Sache her nicht umgehen lässt, wobei die Form so gewählt sein sollte, dass sie nicht verletzend wirkt. Mit anderen Worten, das Phänomen des Taktes und eben auch des physisch-geographischen Taktes kommt auch in dem Gespür dafür zum Tragen, etwas Ungesagtes, Abwesendes, aber Wichtiges in einer angemessenen Form zur Anwesenheit $\mathrm{zu}$ bringen. Hierin liegt der Schlüssel für ein neues Selbstverständnis, eine gegenwärtige Aufgabe Physischer Geographie im starken Sinne - einer anspruchsvollen Geographie, die sich auf ihre Graphizität versteht.

\footnotetext{
${ }^{13}$ Zur Bedeutung des Graphischen bzw. einer Graphizität für die Geographie - gerade auch im Gegensatz zur Derridaschen Auslegung des graphein - vgl. auch Zahnen (2007). Dort habe ich den von mir geprägten Begriff der Geographizität erstmals verwendet, um damit entgegen üblicher Sichtweisen auf die Geographie eine Seinsweise oder Eigenart des Geographischen herausstellen, die sich im Vollzug zeigt und sich übrigens nicht allein auf von Geographen verfasste Texte beschränken muss.
} 
10

\section{Die Darstellungsfrage}

In diesem Zuge komme ich auf „Sprache“ im engeren Sinne zurück, also auf die Darstellungen, die in der Physischen Geographie, besonders in Textform, hervorgebracht werden. In welchem Verhältnis vermögen sie zu der Sprache der irdischen Naturgebilde zu stehen, der eine takt- und anspruchsvolle Geländeerkundung bzw. -erfahrung folgt?

In den Passagen des vorliegenden Aufsatzes, in denen noch keine explizite Trennung zwischen einer physischgeographischen Identität und einer Identität der erdbezogenen Naturwissenschaften vorgenommen wurde, wurden die Darstellungen der Physischen Geographie als naturwissenschaftliche Darstellungen thematisiert: also als gemeinhin sehr explizit und trennscharf formulierte bzw. formalisierende Gesetze, Theorien, Modelle oder Repräsentationen, die im Zuge des Tuns der erdbezogenen Naturwissenschaften in sogenannten ,nomothetischen“ Studien erstellt oder in sogenannten ,idiographischen“ Studien angewendet werden und die sich dadurch auszeichnen, dass die Bedingungen ihrer Gültigkeit bzw. sinnvollen Erstellung oder Anwendung aus den Darstellungen selbst nicht ableitbar sind (s. Abschnitt 3). Anders gesagt: Derart verfasste Darstellungen tragen sich nicht selbst. Daraufhin wurde gezeigt, dass diese Darstellungen, obwohl sie sich nicht selbst tragen, für den Kundigen als gültig bzw. als sinnvoll erstellt oder angewendet und somit als qualitativ wertvoll gelten können, nicht weil sie bloß etablierten Interpretationsmustern eines jeweiligen historisch-kulturellen Kontextes entsprächen, sondern weil sie stillschweigend von der eigenen Geländeerfahrung ergänzt und getragen werden, durch die man sich auf die „Sprache“ der irdischen Naturgebilde versteht, d.h. ihrem tragenden, über etablierte Interpretationsmuster hinausweisenden Anspruch gerecht zu werden vermag (s. Abschnitt 7).

Eine Physische Geographie im starken Sinne hingegen, die auf physisch-geographischem Takt basiert und sich dadurch auch auf die ihr innewohnende Darstellungsproblematik bzw. auf ihre Graphizität versteht, müsste auf andere, weder „rein“ naturwissenschaftliche, noch „rein“ durch die Grenzen eines jeweiligen historisch-kulturellen Kontexts festgelegte (Aspekte von) Darstellungsformen bedacht sein, in denen selbst der Bezug zu der Sphäre eines tragenden Anspruchs der irdischen Naturerscheinungen auf qualitativ wertvolle Weise integriert und gewahrt ist.

Diese Wahrung würde erstens bedeuten, dem eingangs angesprochenen zweifachen Sinn von „Sprache Physischer Geographie“ (bzw. „Geographie“) gerecht zu werden wobei spätestens jetzt deutlich wird, dass es sich bei diesem zweifachen Sinn nicht um ein bloßes Nebeneinander zweier möglicher Bedeutungen von „Sprache Physischer Geographie“ (bzw. „Geographie“) handelt. Das Zwiefache der Sprache Physischer Geographie bedeutet vielmehr, dass die als und in der Geländeerfahrung zum Tragen kommende unausdrückliche "Sprache der irdischen Naturgebilde“ und die „Sprache Physischer Geographen“ (als Sprache Physischer Geographie im engeren Sinne, v.a. in Form von Darstellungen, die Geographen erzeugen) miteinander vermittelt sind. Zweitens würde die Wahrung bedeuten, dass es sich um eine Form von Physischer Geographie handelt, die, anders als die formalisierten naturwissenschaftlichen Darstellungen einer Physischen Geographie im schwachen Sinne, sich selbst zu tragen und somit auch zu revidieren oder neu zu verstehen vermag. Sie bedeutet somit immer auch die Chance der Emergenz einer neuen Form des Geographisch-Seins und somit auch des neuen Schreibens, Zur-Darstellung-Bringens bzw. des Vollzugs von Geographie. Inwiefern dies möglich ist, soll im Folgenden erörtert werden.

Wie sind physisch-geographische Verhältnisse angemessen darzustellen, um sie gewissermaßen vor dem inneren $\mathrm{Au}-$ ge anwesend sein zu lassen? Diese Frage steht nicht nur in einem Bezug zu dem Phänomen des physisch-geographischen Taktes, das ja - wie oben gesagt wurde - auch in dem Gespür dafür zum Tragen kommt, ein Ungesagtes, Abwesendes in einer angemessenen Form zur Anwesenheit zu bringen. Mit der Frage berühren wir auch ein bereits langwährendes Kernproblem der Physischen Geographie, das - zumindest im deutschsprachigen Raum - im Verlauf der Geschichte des Fachs verschiedenste Ausprägungen angenommen hat, aber in der derzeitigen Physischen Geographie eines naturwissenschaftlichen Selbstverständnisses keine explizite Rolle mehr spielt. Können diese verschiedenen Ausprägungen in ihrem fachgeschichtlichen Verlauf hier auch nicht weiter diskutiert werden, so ist doch herauszustellen, dass sich aus dem Gedankengang des vorliegenden Aufsatzes eine revidierte Sicht auf dieses fachhistorisch bekannte Problem ergibt: Denn es kann im Rahmen dieses Aufsatzes weder um die Frage einer korrekten Abbildung eines bestehenden Voroder Urbildes (oder gar Buches der Natur) gehen, noch nur um einen Autor-Text-Leser-Kontext, also darum, eine dem Leser oder Zuhörer angemessene Form zu finden. Stattdessen geht es um einen immer wieder neu zu vollziehenden Darstellungsprozess, der überhaupt erst - und immer wieder neu - in Erfahrung zu bringen ermöglicht, von welcher eigenen Qualität das in der Übergänglichkeit der irdischen Naturerscheinungen gründende, vielschichtige BildungsGebilde eigentlich ist, mit dem man es im „Gelände“ und mit dem man es dann auch in seinen „Darstellungen“ zu tun hat. Auch hier - wie bei der Geländeerkundung - geht es also erneut primär darum, sich in einer übergänglichen evolutiven - Bildungs- und Umbildungsphase zu bewegen. Lässt sich in der Geländeerkundung das leibliche Verhalten des Erkundenden von dem Verhalten bzw. den Verhältnissen des Geländes nicht trennen, so lässt sich auch hier nicht entscheiden, was von wem stammt: die darstellende Sprache von dem sich in einer gewissen Weise darstellenden und darzustellenden übergänglichen irdischen Naturgebilde oder Gelände oder das sich in einer gewissen Weise darstellende und darzustellende irdische Naturgebilde oder Gelände von 
der darstellenden Sprache. Physische Geographie bringt sich so gewissermaßen selbst zur Darstellung.

Nun sind viele Geographen aus eigener Erfahrung mit dem Phänomen vertraut, dass ein Gelände zu beschreiben die Weise verändert, wie sich einem dieses Gelände darstellt, was wiederum die Weise verändert, wie man dieses Gelände beschreibt usw. Auch dies heißt nichts anderes, als dass sich im Zuge dieses Prozesses ein in sich vielschichtiges Bildungs-Gebilde eigener Qualität herausbildet, was weder „rein sprachlich“ noch „rein materiell“ zu verorten ist. Was heißt es jedoch, derartige, sich selbst zur Darstellung bringende übergängliche Gebilde als anspruchsvolle Aufgabe einer Physischen Geographie im starken Sinne zu verstehen, die sich nicht mehr ihren erdbezogenen „Nachbarwissenschaften" (oder gar den naturwissenschaftlichen Grundlagenfächern) anzugleichen sucht und daher auch nicht mehr mit diesen konkurrieren muss, ${ }^{14}$ sondern sich auf ihre Geo-Graphizität versteht und so den Anspruch einer Sphäre der irdischen Naturgebilde in sich auf qualitativ wertvolle Weise wahrt? Und was kann es nicht heißen?

Nicht heißen kann es ein Zurück in die Bemühung, im Gelände „gegebene“ Verhältnisse bloß - also nur oberflächlich und ohne Tragweite - zu beschreiben oder gar zu katalogisieren. Auch nicht heißen kann es ein Vorhaben, das derartige Bildungs- und Umbildungsprozesse anhand von historischen Text-, Bild- oder Kartenmaterialien zu rekonstruieren und festzuhalten versucht.

Um in dieser Hinsicht den entscheidenden Punkt herausstellen zu können, möchte ich zunächst auf ein anderes Beispiel zurückgreifen: die gelebte, lebendige Praxis einer Orientierung im Gelände anhand einer Karte: Auch hier lässt sich nicht eindeutig festlegen, ob die Weise, wie sich das Gelände im Zuge dieses Orientierungsprozesses darstellt, ursprünglich von der Kartendarstellung stammt oder umgekehrt die Kartendarstellung sich von der Orientierung im Gelände verstehen lässt. Zwar könnte man - wie übrigens auch bei den in Textform vorliegenden Geländebeschreibungen, wenn dort auch weniger wahrscheinlich - in der Rolle eines distanzierten Beobachters einer solchen Praxis versucht sein, diesen Wunsch nach eindeutigen Festlegungen durch die Erstellung von chronologischen Nachweisen befriedigen $\mathrm{zu}$ wollen, etwa derart, wie es bereits eine historische Forschung zu Kartenproduktionsprozessen gibt, die Schritt für Schritt nachzuweisen und abzusichern sucht, warum eine Karte wann, wie und von wem erstellt oder geändert wurde. Doch so sinnvoll ein solches Vorgehen für bestimmte Kontexte sein mag, es hieße, die Aufgabe, um die es mir hier geht, zu verkennen. Nicht nur, weil dabei vergessen würde, dass wir nicht voraussetzungslos sind, weil wir in der Sprache wie auch in den Naturgebilden der Erde -

\footnotetext{
${ }^{14} \mathrm{Zu}$ den Schwierigkeiten, die ein derartiges Konkurrieren mit sich bringt, vgl. dazu auch Zahnen (2003) sowie die Diskussion des von Frodeman (1995) aufgebrachten Begriffs des ,physics envy“ in Massey (1999).
}

und somit auch in der Sprache Physischer Geographie im genannten zweifachen Sinne - immer schon sind, was ein Chronologisierungsvorhaben nur bedingt hilfreich sein lässt. Auch und zugleich, weil es etwas vollkommen anderes ist, als distanzierter Beobachter einen „Bildungs- und Umbildungsprozess" analysieren zu wollen - wobei es kaum noch angemessen erscheint, dann noch von Bildung und Umbildung zu sprechen - oder selbst in diesen involviert zu sein, ja sich mit dieser Situation geradezu zu identifizieren, wie es eben z.B. in der Situation einer notwendigen Orientierung im Gelände geschieht. ${ }^{15}$ Nur aus einer eigens gelebten und immer wieder neu zu erlebenden Kontextualität, in der man sich seine eigene gegenwärtige Situation überhaupt erst bzw. wieder neu erschließt, und nicht aus der Position eines distanzierten Beobachters, der die konkreten Bildungs- und Umbildungsprozesse anderer beschreiben, rekonstruieren, analysieren oder in Materialien wiederfinden und darüber schreiben will, gibt es die Möglichkeit, einem über bisherige Darstellungen immer hinausweisenden tragenden Anspruch der Sache zu folgen - im zweifachen Sinne der Sprache Physischer Geographie. Das heißt, dass die anvisierte Aufgabe der Physischen Geographie gerade darin besteht, unsere gegenwärtige physisch-geographische Situation - die natürlich auch historisch-kulturell bedingt ist, aber eben über historisch-kulturelle Bedingtheiten hinausweisend immer in eine Sphäre des Anspruchs der irdischen Naturerscheinungen hineinragt - immer wieder zur Frage werden und überhaupt erst bzw. wieder neu zur Darstellung kommen zu lassen, woraus sich auf unumkehrbare Weise Aufgaben einer neuen Qualität ergeben. Dies kann auf zweierlei Weise geschehen:

Zum einen dadurch, dass auf der Basis eines eigenen, evolutiven Erlebens im Gelände Naturgebilde der Erde in ihrer eigenen Qualität, Übergänglichkeit und Vielschichtigkeit in einer Weise neu bzw. überhaupt erst beschrieben oder dargestellt werden, die das Darstellungsvermögen der Physischen Geographie im zweifachen Sinne erweitert: also nicht nur das Darstellungsvermögen der Geographen, sondern ebenso die aus den gewonnenen Darstellungen resultierende Öffnung für das Darstellungsvermögen, die „Sprache“ der irdischen Naturerscheinungen. Dabei ist dieser Erweiterungsprozess insofern unumkehrbar, als er, einmal eigens vollzogen, zu differenzierteren bzw. vielschichtigeren Ansprüchen an Erkundungen und Darstellungen führt. Würde man diesen nicht nachkommen, bliebe ein Gefühl der Unstimmigkeit, Unangemessenheit oder Unabgerundetheit. Darin zeigt sich,

\footnotetext{
${ }^{15}$ Womit ich nicht sagen will, dass diejenigen, die sich in der Rolle eines distanzierten Beobachters wähnen, nicht auch einem solchen Identifikationsprozess unterliegen können - im Gegenteil, ihr persönliches Interesse an bestimmten Gegenständen hat mit einem solchen Prozess bereits zu tun. Die Frage ist nur, ob sich dies durch die Überwindung der analytischen Distanz auch in der Form ihres Tuns bzw. ihrer Darstellungen in einer Weise niederschlägt, die - im „Gespräch“ mit der Sache - zu einer Vertiefung des eigenen Anspruchs führt, der dadurch weiter tragen kann.
} 
dass es sich bei dem Erweiterungsprozess um ein Geschehen handelt, das eine kairetische Gegenwart, einen Augenblick eines ,Jetzt-so-und-so-nie-wieder“"16 durchläuft, genauer: eines Jetzt-so-Geographie-und-so-nie-wieder.

Zum anderen - und in gewisser Weise zugleich - kann dies dadurch geschehen, dass anhand von Materialien, die - egal wann oder wo - von Geländeerfahrenen bzw. Geländekundigen auf der Basis eines tragenden Anspruchs der irdischen Naturgebilde verfasst wurden, brachliegende Potenziale des geographischen Fachgebiets auf eine Weise aufgedeckt und zur Darstellung gebracht werden, die ebenfalls evolutiv bzw. von geographischem Takt geleitet ist und somit selbst in Form der Herausbildung eines neuen, in sich übergänglichen, vielschichtigen, geographischen (Text-)Gebildes eigener Qualität geschieht. Dabei handelt es sich dann um geographische (Text-)Gebilde, die uns über unsere Geographizität und somit die Eigenart eines (Sich-)Darstellens von Geographie neu ins Bild kommen lassen und daher uns wieder neue, aber dem Fach ureigene Aufgaben zu erschließen vermögen, die man fortan nicht mehr ignorieren kann. Auch in diesem Prozess, in dem geographische Materialien sowohl Ausdruck als auch Aufschluss physisch-geographischer Situationen sind, wird eine kairetische Gegenwart im Sinne eines Jetzt-so-Geographie-und-so-nie-wieder erfahren.

Auf beide - miteinander zusammenhängende - Weisen wird Physische Geographie vollzogen, indem man sie neu vollzieht, und d.h.: indem man sich neu auf sie versteht in einer Weise, hinter die man, wenn man anspruchsvoll ist und sein Fach weiterbringen will, nicht zurückkann. Geographie in einem starken Sinne - und das heißt auch: Geographie als ein eigenständiges Fachgebiet - gibt es nur in diesem Prozess, in dem sich die eigene geographische Situation bzw. das geographische Fachgebiet selbst neu herausbildet, zu einem neuen Gebilde neuer Möglichkeiten wird. Geographie kann daher vollzogen werden, indem die öffnenden, ins Bild setzenden Potenziale zur Darstellung gebracht und daher gewahrt werden, die Naturgebilden der Erde, aber auch physisch-geographischen Materialien in textlicher oder anderer Form auf verborgene, aber eben $\mathrm{zu}$ entbergende - oder erschließende - Weise innewohnen können. ${ }^{17}$ Anders gesagt: Physische Geographie, ja Geographizität wird vollzogen, und sie trägt sich selbst, indem sie - geographie-schreibend - einem Anspruch der irdischen „Materialitäten“ nicht nur im Feld, sondern auch in den Materialien des Fachs folgt:

Denn die Materialien sind natürlich nicht allein Materialien einer Sprache der Physischen Geographie im engeren Sinne. Gefolgt wird primär immer einem Anspruch der Sphäre der irdischen Naturerscheinungen selbst - also

\footnotetext{
${ }^{16}$ Die Formulierung ,Jetzt-so-und-so-nie-wieder“ übernehme ich aus Gadamer und Stappert (2002).

${ }^{17}$ Kennern der Heideggerschen oder auch Gadamerschen Philosophie wird an dieser Stelle der Bezug zum aletheischen Wahrheitsbegriff nicht verborgen bleiben.
}

gewissermaßen einem Anspruch der „Materialitäten“, einem Anspruch der Physis: Die Darstellungen einer Sprache der Physischen Geographie im engeren Sinne können nämlich gerade dann für uns anspruchsvoll sein (und uns daher wieder neu ins Bild kommen lassen) wenn sie auf der Basis der leiblichen Erfahrung irdischer Naturerscheinungen in ihrer eigenen Qualität aus einem Prozess der im obigen Sinne taktvollen (Text- bzw. Darstellungs-)Formsuche und Formfindung hervorgegangen sind und somit in ihnen eine Sphäre des Ungesagten, Unausdrücklichen oder abwesend Anwesenden mitschwingt, statt aus einer durch und durch definierten und definierbaren bzw. formalisierbaren Sprache zu bestehen. Dadurch wird uns der Vollzug von Geographie neu ermöglicht. Wenn man so will, ist schon die zu Beginn des vorliegenden Aufsatzes erwähnte, von geländeerfahrenen Wissenschaftlern gewählte (weil der Sache nach als angemessen empfundene) Formulierung eines „Gesprächs mit der Erde" ein Beispiel für eine solche uns ins Bild kommen lassende „Darstellung“, wie die im Gedankengang dieses Aufsatzes entfalteten Potenziale dieser Formulierung zeigen. Sie ermöglicht uns, Physische Geographie - und uns auf Physische Geographie - auf unumkehrbare Weise neu zu verstehen. Darin liegt $i h r$ welterschließendes, ins Bild setzendes, die eigene physisch-geographische Situation überhaupt erst zur Darstellung bringendes Potenzial.

\section{Ausblick}

Zwar meinten die Physischen Geographinnen und Geographen im Zuge der Szientifizierung ihres Fachs Darstellungen einer derartigen Qualität - und somit einer bildlichen Sprache - gerade preisgeben zu müssen, ebenso wie geographischen Takt. Doch würden sie an dieser Entscheidung festhalten, verbauten sie sich erstens die Möglichkeit einer eigenständigen Positionierung und Identität gegenüber den erdbezogenen Naturwissenschaften und zugleich die Möglichkeit, diese Wissenschaften auf genuin geographische, grundlegende Weise zu befruchten: Denn schließlich müssen sich auch diese Wissenschaften, um qualitativ ernstzunehmen zu sein, immer wieder neu ins Bild setzen; d.h. sie können gerade durch die Entfaltung des Potenzials einer solchen Sprache bzw. solcher Darstellungen, in denen auf der Basis geographischen Takts - und somit aus einer Sphäre des Unverstandenen, neu zu Verstehenden - Unausdrückliches mitschwingt, an Qualität gewinnen. Die Physischen Geographinnen und Geographen verbauten sich zweitens auch die Möglichkeit, ein aus ihrer physischgeographischen Situation erwachsendes, neues und eigenes Licht auf sozial- oder kulturwissenschaftliche Ansätze zu werfen, die - ob in der Wissenschaftsforschung, in den area studies oder anderswo - die sogenannte „materielle“ Dimension, die Dimension der „Natur“, einzubeziehen suchen oder suchen könnten: Denn schließlich sind die „Gegenstände“ dieser Wissenschaften immer schon und somit unauflöslich, 
jedoch ohne dass dies gemeinhin thematisch würde, in physisch-geographischen Verhältnissen eigenen Anspruchs situiert. Schließlich aber verbauten sie sich - drittens - die Chance, die Dichotomie zwischen der Humangeographie und der Physischen Geographie aufbrechen zu können sowie - viertens - zu den Materialien ihrer eigenen Geschichte wieder einen Zugang zu bekommen: Denn indem wir die Sprache Physischer Geographie nicht als ein zu definierendes Mittel, sondern als ein Medium ${ }^{18}$ zu verstehen lernen, das Ausdruck und Aufschluss anspruchsvoller physischgeographischer Situationen sein kann, wird Physische Geographie auch zu einer Frage unserer eigenen Physis, unserer Leiblichkeit und unseres Gespürs, das heißt in einem umfassenden Sinne auch unserer eigenen, menschlichen Medialität. Und indem uns diese vielschichtige Medialität in die Lage versetzt, neue Welten zu erschließen, erschließen sich - wie sich in dem vorliegenden Aufsatz angedeutet hat - zugleich und notwendig auch Grundprobleme der eigenen Fachgeschichte, die, so sehr das Fach auch in eine Vielzahl von Einzelwissenschaften auseinanderzufallen scheint, bis heute weiterhin zum Tragen kommen.

Acknowledgement. Die Autorin dankt der Deutschen Forschungsgemeinschaft für die finanzielle Förderung ihres Forschungsprojektes zum ,Verstehen der Erdnatur“, aus dem auch der vorliegende Aufsatz hervorgegangen ist.

Edited by: A. Schlottmann

\section{Literatur}

Ahnert, F.: Einführung in die Geomorphologie, Ulmer, Stuttgart, 1996.

Baker, V. R.: Conversing with the earth: The Geological Approach to understanding, in: Earth Matters. The Earth Sciences, Philosophy and the Claims of the Community, edited by: Frodeman, R., Prentice-Hall, Upper Saddle River, 2-10, 2000.

Blasche, S., Gutmann, M., und Weingarten, M. (Eds.): Repraesentatio Mundi. Bilder als Ausdruck und Aufschluss menschlicher Weltverhältnisse, Historische und systematische Perspektiven, Bielefeld, 2004.

Cloos, H.: Gespräch mit der Erde. Welt- und Lebensfahrt eines Geologen, Frankfurt a.M., 1968.

Crease, R.: The play of nature. Experimentation as performance, The Indiana Series in the Philosophy of Technology, Indiana University Press, Bloomington, 1993.

Crease, R.: Hermeneutics and the natural sciences: Introduction, Man and World, 30, 3, 259-270, 1997.

Demeritt, D. and Dyer S.: Dialogue, metaphors of dialogue and understandings of geography, Area, 34, 3, 229-241, 2002.

Eger, M.: Language and the Double Hermeneutic in Natural Science, in: Hermeneutics and Science, Boston Studies in the Philosophy of Science, 206, edited by: Fehér, M., Kiss, O., and Ropolyi, L., 265-280, 1999.

\footnotetext{
${ }^{18}$ Zur Problematik des Verhältnisses von Mittel und Medium vgl. auch Blasche et al. (2004).
}

Fehér, M., Kiss, O., and Ropolyi, L. (Eds.): Hermeneutics and Science, Boston Studies in the Philosophy of Science, 206, 1-377, 1999.

Frodeman, R.: Geological reasoning: Geology as an interpretive and historical science, Geol. Soc. Am. Bull., 107, 8, 960-968, 1995.

Gadamer, H.-G.: Die Natur der Sache und die Sprache der Dinge, Gesammelte Werke, 2, Tübingen, 66-76, 1999a (first 1960).

Gadamer, H.-G.: Text und Interpretation, Gesammelte Werke, 2, Tübingen, 330-360, 1999b (first 1983).

Gadamer, H.-G.: Wahrheit und Methode. Grundzüge einer philosophischen Hermeneutik, Gesammelte Werke, 1, Tübingen, 1999c (zuerst 1960).

Gadamer, H.-G. und Stappert, B. H.: Kairos - Über die Gunst des Augenblicks und das weise Maß, Sinn und Form, 54, 2, 149-160, 2002.

Ginev, D.: Essays in the Hermeneutics of Science, Avebury Series in Philosophy, Aldershot, 1997.

Ginev, D.: On the hermeneutic fore-structure of scientific research, Cont. Philos. Rev., 32, 143-168, 1999.

Ginev, D.: The Hermeneutic Context of Constitution, in: Hermeneutic Philosophy of Science, Van Gogh's Eyes, and God, edited by: Babich, B., Dordrecht, 43-52, 2002.

Heelan, P. A.: Natural Science as a hermeneutic of instrumentation, Philos. Sci., 50, 181-204, 1983.

Heelan, P. A.: Yes! There Is a Hermeneutics of Natural Science: A Rejoinder to Markus, Sci. Context, 3, 2, 477-488, 1989.

Heelan, P. A.: Galileo, Luther, and the hermeneutics of natural science, in: The questions of hermeneutics, edited by: Stapleton, T. J., Dordrecht, 363-375, 1994.

Heelan, P. A.: The scope of hermeneutics in natural science, Studies in the History and Philosophy of Science, 29, 2, 273-298, 1998.

Heelan, P. A.: Phenomenology and the philosophy of the natural sciences, in: Phenomenology World-Wide (Encyclopedia: Phenomenology Foundations: A Guide for Research and Study), edited by: Tymieniecka, A.-T., Dordrecht, 631-641, 2002.

Hogrebe, W.: Hermes und Apoll: Gadamers Universalitätsanspruch der Hermeneutik, in: Ästhethik - Hermeneutik - Neurowissenschaften, herausgegeben von: Breidbach, O. und Orsi, G., Münster, 19-30, 2004.

Kisiel, T. J.: A hermeneutics of natural sciences? The debate updated, Man World, 30, 3, 329-342, 1997.

Kockelmans, J. J.: On the hermeneutical nature of modern natural science, Man World, 30, 3, 299-313, 1997.

Kockelmans, J. J. and Kisiel, T. J.: Phenomenology and the Natural Sciences, Northwestern University Press, Evanston, 1970.

Latour, B.: Science in action, Harvard Universtity Press, Cambridge, 1987.

Lippuner, R.: Raum, Systeme, Praktiken: zum Verhältnis von Alltag, Wissenschaft und Geographie, Sozialgeographische Bibliothek, 2, Stuttgart, 2005.

Massey, D.: Space-time, 'science' and the relationship between physical geography and human geography, T. I. Brit. Geogr., 24, 261-276, 1999.

Phillips, J. D.: Methodology, Scale, and the Field of Dreams, Ann. Assoc. Am. Geogr., 89, 4, 754-760, 1999.

Pickering, A.: The mangle of practice. Time, agency and science, University of Chicago Press, Chicago, 1995. 
Rheinberger, H.-J.: Historische Epistemologie zur Einführung, Hamburg, 2007.

Thornes, J. B.: Evolutionary Geomorphology, Geography, 68, 225235, 1983

Zahnen, B.: Anwendende Klimatologie zwischen Daten und Deutung, Alltagswelt und Klimaphysik. Überlegungen entwickelt am Beispiel einer stadtklimatologischen Studie des Flugfelds Berlin-Tempelhof, Mensch \& Buch Verlag, Berlin, 2003.

Zahnen, B.: Fragwürdigkeit und Eigensinn der Geographie, Geogr. Z., 93, 4, 201-220, 2005.
Zahnen, B.: Im Auge der Physischen Geographie. Vom Blicken und Sehen, Erdkunde, 60, 3, 231-245, 2006.

Zahnen, B.: Lesen, Zeitlichkeit und das Geographische der Physischen Geographie, Geogr. Z., 95, 1+2, 72-90, 2007.

Zahnen, B.: Das implizite Wissen der Erdwissenschaftler. Ein Beitrag zur Ästhetik und Geschichtlichkeit Physischer Geographie, Berichte zur Deutschen Landeskunde, 82, 2, 173-190, 2008a.

Zahnen, B.: Schleichende Risiken als geographisches Problem der Zeit. Ein Beitrag zur Theorie der Geographie, Geographische Revue, 10, 1, 15-29, 2008 b. 